\section{Fatores associados a distúrbios do sono em estudantes universitários}

\author{
Factors associated with sleep disorders in \\ university students
}

\section{Factores asociados a trastornos del sueño en estudiantes universitarios}

\section{Resumo}

O objetivo foi investigar distúrbios do sono e fatores sociodemográficos e comportamentais associados. Foi realizado um censo com universitários. Perguntas extraídas do Munich Chronotype Questionnaire investigaram: duração insuficiente do sono ( $<6$ horas/dia para $<65$ anos $e<5$ horas/dia para os demais), latência longa (> 30 minutos), baixa qualidade autopercebida, despertares noturnos (involuntários, no meio da noite) e sonolência diurna (dificuldade de concentração). Variáveis independentes englobaram caracteristicas sociodemográficas e comportamentais. Análises ajustadas foram feitas com regressão de Poisson. Dos 1.865 estudantes, 32\% apresentaram sono insuficiente nos dias de aula, 8,2\% sono insuficiente nos finais de semana, 18,6\% latência longa nos dias de aula, 17,2\% latência longa nos finais de semana, $30 \%$ baixa qualidade autopercebida, 12,7\% despertares noturnos e 32,2\% sonolência diurna. O maior consumo de álcool esteve associado à duração insuficiente e latência longa nos dias de aula, baixa qualidade, despertares e sonolência; tabagismo com duração insuficiente nos finais de semana, despertares e baixa qualidade; e cor da pele preta ou parda com duração insuficiente nos dias de aula, baixa qualidade e despertares. Ter aulas de manhã associou-se a sono insuficiente nos dias de aula e sonolência diurna, e o sexo feminino, sonolência, baixa qualidade e despertares. Os distúrbios de sono mais frequentes foram sono insuficiente nos dias de aula, baixa qualidade autopercebida e sonolência diurna. O consumo de álcool e cigarros e a cor preta ou parda foram os principais fatores associados aos distúrbios.

Distúrbios do Sono; Sono; Sonolência; Estudantes
Caroline Maria de Mello Carone 1

Bianca Del Ponte da Silva 1

Luciana Tovo Rodrigues 1

Patrice de Souza Tavares 1

Marina Xavier Carpena 1

Iná S. Santos 1

doi: 10.1590/0102-311X00074919

\section{Correspondência}

C. M. M. Carone

Universidade Federal de Pelotas.

Rua Marechal Deodoro 1160, Pelotas, RS 96020-220, Brasil.

carolinemcarone@yahoo.com.br

1 Universidade Federal de Pelotas, Pelotas, Brasil. 


\section{Introdução}

O sono é um fenômeno vital, durante o qual se "desligam” ou atenuam-se mecanismos e sistemas orgânicos, com vistas à prevenção da exaustão, e são executados processos de recuperação e compensação de gastos energéticos e bioquímicos, ocorridos no período de atividade ${ }^{1}$. Distúrbios do sono podem resultar em repercussões negativas para o ser humano, como déficit cognitivo, alterações no metabolismo e distúrbios psicológicos 2. A má qualidade do sono e o sono insuficiente são fatores associados a patologias, como obesidade, distúrbios mentais, hipertensão arterial e diabetes 3 .

O sono tem importantes funções biológicas na consolidação da memória, termorregulação e restauração do metabolismo energético cerebral 4. Devido à má conservação dessas funções, estudantes que não mantêm uma qualidade e quantidade de sono adequadas podem ter seu processo de aprendizagem e desempenho acadêmico prejudicados 5,6. Adolescentes com duração insuficiente do sono apresentam maior frequência de obesidade, doença cardiovascular e mortalidade por todas as causas, bem como maior prevalência de depressão, ansiedade, déficit de atenção, problemas de conduta, uso de drogas e álcool, baixo desempenho escolar e pensamentos suicidas, além de sintomas como cefaleia, dores ligadas ao aparelho digestivo e lombalgia 7,8,9.

Dentre os fatores associados aos distúrbios do sono entre estudantes estão as características sociodemográficas e comportamentais 7,8,9,10. O conhecimento sobre a epidemiologia dos distúrbios do sono poderá auxiliar no planejamento de ações que visem a qualificar o sono dos estudantes. Assim, este estudo objetiva investigar distúrbios do sono e fatores sociodemográficos e comportamentais associados, entre estudantes de uma universidade pública federal no sul do Brasil.

\section{Metodologia}

A Universidade Federal de Pelotas (UFPel) é uma instituição de ensino localizada em Pelotas, cidade de porte médio do Sul do Brasil. Anualmente, a UFPel recebe cerca de 3 mil novos alunos, distribuídos em seus 83 cursos presenciais de graduação.

Foi realizado um censo entre os estudantes com 18 anos ou mais de idade, considerando como critério de elegibilidade ter ingressado na UFPel em 2017, e estar matriculado em disciplinas do segundo semestre do mesmo ano, nos respectivos cursos presenciais da universidade. Os dados foram coletados em questionários autoaplicados, respondidos pelos alunos em sala de aula, utilizando-se tablets com o sistema RedCap 11, sendo deslocada para aplicação uma equipe de no mínimo dois mestrandos. O trabalho de campo iniciou em novembro/2017 e foi concluído em julho/2018.

Os desfechos avaliados foram sono insuficiente nas 24 horas em dias de aula e nos finais de semana, tempo de latência do sono noturno em dias de aula e nos finais de semana, qualidade autopercebida do sono, ocorrência de despertares noturnos e sonolência diurna.

\section{Sono insuficiente em dias de aula e finais de semana}

A duração do sono nas 24 horas, em dias de aula, foi obtida por meio das perguntas "Nos dias de aula, eu realmente estou pronto(a) para dormir às __ horas __ minutos", "Necessito de __ minutos para adormecer" e “Acordo às__ horas__ minutos”, extraídas da versão em português 12 do Munich Chronotype Questionnaire (MCTQ) 13 .

Da mesma forma, foi obtida a duração do sono nas 24 horas dos finais de semana, somente substituindo no questionário os "dias de aula" por "dias de final de semana". O número de horas de sono foi calculado pela diferença entre o horário em que o indivíduo acorda e o horário em que realmente está pronto para dormir, subtraindo-se os minutos que necessita para adormecer (tempo de latência). A duração insuficiente do sono foi definida conforme o ponto de corte recomendado pela Fundação Nacional do Sono (Estados Unidos), de acordo com a idade 14: 7-9 horas para indivíduos entre 18 e 64 anos de idade (limites aceitáveis de 6-11 horas/dia) e 7-8 horas para idosos (65 anos ou mais) (limites aceitáveis 5-9 horas/dia). Dessa forma, considerou-se duração insuficiente o sono de 24 horas inferior a 6 horas/dia para adultos de 18-64 anos e inferior a 5 horas/dia para indivíduos com 65 anos ou mais. 
O tempo de latência foi obtido da resposta à pergunta: "Necessito de _- minutos para adormecer", extraída da versão em português 12 do MCTQ 13. Tempos superiores a 30 minutos foram classificados como períodos longos de latência 15 .

\section{Qualidade autopercebida do sono}

A qualidade autopercebida foi obtida mediante a resposta, em escala Likert (muito boa, boa, regular, ruim, péssima), à pergunta: "De modo geral, como você avalia a qualidade do seu sono nos últimos 30 dias?". Estudantes que responderam "ruim" ou "péssima” foram classificados como apresentando sono de baixa qualidade.

\section{Despertares noturnos}

Os despertares noturnos foram obtidos por meio da resposta à pergunta: "Nas últimas quatro semanas, voce acordou de madrugada e teve dificuldade para voltar a dormir?", com as seguintes opções de resposta: "nunca, de vez em quando, na maioria das noites ou sempre". Aqueles que responderam "na maioria das noites" ou "sempre" foram classificados como apresentando despertares noturnos.

\section{Sonolência diurna}

A sonolência diurna foi obtida mediante a resposta à pergunta: "Nas últimas quatro semanas, você sentiu sonolência que atrapalhava para assistir às aulas?", com as seguintes opções de resposta: "nunca, de vez em quando, na maioria dos dias ou sempre", sendo consideradas positivas as respostas "na maioria dos dias" ou "sempre".

Como variáveis independentes, foram investigadas as características sociodemográficas e comportamentais: sexo; idade (em anos); cor autorreferida (branca, parda ou preta); nível econômico da família, obtido de acordo com o Critério de Classificação Econômica Brasil, da Associação Brasileira de Empresas de Pesquisa (ABEP - http://www.abep.org/criterio-brasil), cujas perguntas são referentes aos bens da família, mesmo que o estudante morasse sozinho, e categorizado em classes A (mais alta), B, C, D-E; número de pessoas que residiam na casa com o estudante; número de pessoas com quem compartilhava o quarto; número de pessoas com quem compartilhava a cama; turno do curso (manhã, tarde, noite ou integral); número habitual de horas de uso de tela (computador, videogame, televisão e outras mídias) nas 24 horas, posteriormente categorizado em tercis; número de horas semanais de prática de atividade física, coletado com o Questionário Internacional de Atividade Física (IPAQ) 16, sendo os estudantes posteriormente classificados em inativos ( $<300$ minutos semanais) e ativos $(\geq 300$ minutos semanais); tabagismo atual (sim ou não, sendo se fumou pelo menos um cigarro por dia, nos últimos 30 dias); frequência de consumo de bebidas alcoólicas, de acordo com o Alcohol Use Disorder Identification Test (AUDIT) 17, que classifica os indivíduos com consumo nocivo de álcool quando a pontuação é igual ou superior a 8 18; e curso no qual o estudante estava matriculado, sendo, posteriormente, categorizado em quatro grandes áreas (Ciências Exatas e da Terra/Agrárias e Engenharias, Ciências da Saúde e Biológicas, Ciências Sociais Aplicadas e Humanas, e Linguística, Letras e Artes).

As análises dos dados foram realizadas no programa Stata versão 15.0 (https://www.stata.com). A descrição dos dados foi realizada por meio do cálculo de medidas de tendência central e proporções com intervalos de 95\% de confiança (IC95\%), para variáveis categóricas. As análises de associação foram feitas com os desfechos na forma categórica. Foram calculadas as prevalências dos desfechos e razões de prevalências (RP) brutas e ajustadas, com seus respectivos IC95\% por regressão de Poisson. Para cada desfecho, foi realizada análise bruta com cada uma das variáveis independentes, sendo levadas para a análise ajustada aquelas que se associaram com valor de $\mathrm{p} \leq 0,20$. Foram consideradas estatisticamente significativas as associações que apresentaram valor de $\mathrm{p}<0,05$ após ajuste para as demais variáveis. $\mathrm{Na}$ análise multivariável, foram mantidas no modelo, para fins de ajuste, as variáveis associadas ao desfecho com $\mathrm{p} \leq 0,20$. Os indivíduos sem informação para algum desfecho foram excluídos somente das análises do respectivo desfecho. 
O protocolo do estudo foi aprovado pelo Comitê de Ética em Pesquisa da Faculdade de Medicina da UFPel (Parecer número 2.352.451). Os estudantes assinaram um Termo de Consentimento Livre e Esclarecido, em que ficava garantido o sigilo das informações coletadas e o direito de interromper a participação no trabalho, a qualquer momento, sem ônus de qualquer natureza.

\section{Resultados}

Dentre os ingressantes na UFPel no primeiro semestre de 2017, estavam matriculados no segundo semestre daquele ano um total de 2.706 alunos com 18 anos ou mais de idade. Desses, 623 (23\%) trancaram a matrícula ou abandonaram o curso antes de serem abordados pela pesquisa, $49(1,8 \%)$ se recusaram a participar do estudo e 169 (6,3\%), embora continuassem matriculados, não foram encontrados após pelo menos duas tentativas de busca em dias e horários diferentes. Restaram 1.865 (69,5\% dos elegíveis) para a atual análise. As perdas e recusas foram mais frequentes entre estudantes do sexo masculino $(34,3 \%)$ do que entre os do feminino $(27,7 \%)(\mathrm{p}<0,001)$ e entre os alunos das áreas das Ciências Exatas e da Terra/Agrárias e Engenharias (36,9\%), em comparação aos estudantes das Ciências Sociais Aplicadas e Humanas (31,1\%), Linguística, Letras e Artes (27,7\%) e Ciências da Saúde e Biológicas $(21,5 \%)$ ( $\mathrm{p}<0,001)$. Além disso, o maior percentual de perdas ocorreu entre os estudantes com 23 anos ou mais (44,5\%) em comparação aos de 20-22 anos (28,5\%) e aos de 18-19 anos (20,7\%).

As características sociodemográficas e comportamentais dos estudantes são apresentadas na Tabela 1. A maioria dos participantes era do sexo feminino (54,8\%) e de cor branca $(73,3 \%)$. A maior proporção dos estudantes $(41,4 \%)$ tinha entre 18 e 19 anos de idade e pertencia à classe social B (44,2\%). Mais de 90\% compartilhavam a casa onde moravam, sendo que destes, 7,1\% moravam com mais de cinco pessoas; $37,7 \%$ compartilhavam o quarto de dormir; e 25,3\% a cama. Mais da metade (55\%) declararam-se fisicamente ativos, $11 \%$ eram tabagistas, 33,3\% apresentavam consumo nocivo de bebidas alcoólicas e cerca de 30\% reportaram entre 5 e 16,5 horas de uso de tela por dia (limites do maior tercil). Quanto ao horário das aulas, a maioria frequentava o turno da manhã $(57,2 \%)$ e mais de um terço estava matriculado em cursos da área de Ciências Exatas e da Terra/Agrárias e Engenharias $(34,4 \%)$.

Tabela 1

Prevalências de sono insuficiente e latência longa, em dias de aula e em finais de semana, segundo características sociodemográficas e comportamentais de estudantes universitários $(n=1.865)$.

\begin{tabular}{|c|c|c|c|c|c|}
\hline Características & n (\%) & $\begin{array}{c}\text { Sono insuficiente } \\
\text { nos dias de aula } \\
\%(\text { IC95\%) }\end{array}$ & $\begin{array}{c}\text { Sono insuficiente } \\
\text { nos finais de } \\
\text { semana } \\
\%(\text { IC95\%) }\end{array}$ & $\begin{array}{c}\text { Latência }>30 \\
\text { minutos em dias } \\
\text { de aula } \\
\%(\text { IC95\%) }\end{array}$ & $\begin{array}{c}\text { Latência > } 30 \\
\text { minutos em finais } \\
\text { de semana } \\
\%(\text { IC95\%) }\end{array}$ \\
\hline Total & $1.865(100,0)$ & $31,8(29,6-33,9)$ & $8,2(6,9-9,5)$ & $18,6(16,8-20,6)$ & $17,2(15,4-19,0)$ \\
\hline \multicolumn{6}{|l|}{ Sexo } \\
\hline Masculino & $841(45,2)$ & $34,1(30,9-37,3)$ & $9,8(8,0-12,0)$ & $17,2(14,7-20,1)$ & $18,3(16,0-20,9)$ \\
\hline Feminino & $1.021(54,8)$ & $30,0(27,2-32,8)$ & $6,8(5,4-8,5)$ & $19,9(17,4-22,6)$ & $15,8(13,4-18,5)$ \\
\hline \multicolumn{6}{|l|}{ Cor da pele } \\
\hline Branca & $1.343(73,3)$ & $29,8(27,4-32,3)$ & $7,3(6,1-8,9)$ & $17,5(15,4-19,8)$ & $16,3(14,3-18,4)$ \\
\hline Parda & $247(13,5)$ & $36,9(31,0-43,1)$ & $9,4(6,3-13,8)$ & $23,7(18,5-29,9)$ & $19,2(14,6-24,7)$ \\
\hline Preta & $242(13,2)$ & $37,2(31,3-43,6)$ & $10,5(7,2-14,0)$ & $19,3(14,5-25,3)$ & $19,6(14,9-25,3)$ \\
\hline \multicolumn{6}{|l|}{ Idade (anos) } \\
\hline $18-19$ & $768(41,5)$ & $33,3(30,1-36,8)$ & $7,0(5,4-9,1)$ & $17,1(14,5-20,1)$ & $17,5(14,9-20,5)$ \\
\hline $20-22$ & $603(32,5)$ & $29,4(25,8-33,1)$ & $6,8(5,0-9,1)$ & $19,9(16,7-23,5)$ & $15,6(12,9-18,8)$ \\
\hline$\geq 23$ & $481(26,0)$ & $32,2(28,2-36,5)$ & $11,6(9,1-14,8)$ & $19,6(16,0-23,7)$ & $18,3(15,0-22,1)$ \\
\hline
\end{tabular}

(continua) 
Tabela 1 (continuação)

\begin{tabular}{|c|c|c|c|c|c|}
\hline Características & n (\%) & $\begin{array}{l}\text { Sono insuficiente } \\
\text { nos dias de aula }\end{array}$ & $\begin{array}{l}\text { Sono insuficiente } \\
\text { nos finais de } \\
\text { semana }\end{array}$ & $\begin{array}{c}\text { Latência > } 30 \\
\text { minutos em dias } \\
\text { de aula }\end{array}$ & $\begin{array}{c}\text { Latência > } 30 \\
\text { minutos em finais } \\
\text { de semana }\end{array}$ \\
\hline & & $\%$ (IC95\%) & $\%$ (IC95\%) & \% (IC95\%) & \% (IC95\%) \\
\hline \multicolumn{6}{|l|}{ Classe social } \\
\hline$A$ & $266(14,9)$ & $29,7(24,5-35,5)$ & $5,6(3,4-9,2)$ & $20,7(16,0-26,3)$ & $16,0(11,9-21,0)$ \\
\hline B & $787(44,2)$ & $31,5(28,3-34,8)$ & $8,0(6,3-10,1)$ & $17,2(14,6-20,1)$ & $16,7(14,2-20,0)$ \\
\hline C & $649(36,5)$ & $33,6(30,1-37,4)$ & $9,6(7,6-12,1)$ & $20,0(16,9-23,5)$ & $17,6(14,8-20,8)$ \\
\hline D-E & $78(4,3)$ & $36,4(26,3-47,8)$ & $7,8(3,5-16,5)$ & $13,0(6,8-23,4)$ & $20,5(12,7-31,5)$ \\
\hline \multicolumn{6}{|l|}{ Compartilha a casa onde mora } \\
\hline Não & $182(9,8)$ & $28,3(22,1-35,4)$ & $5,0(2,6-9,4)$ & $17,3(12,2-24,0)$ & $17,5(12,5-24,0)$ \\
\hline Sim & $1.680(90,2)$ & $32,1(29,9-34,3)$ & $8,5(7,3-9,9)$ & $18,8(16,8-20,8)$ & $17,1(15,4-19,1)$ \\
\hline \multicolumn{6}{|l|}{ Compartilha o quarto onde dorme } \\
\hline Não & $1.155(62,3)$ & $31,7(29,1-34,5)$ & $7,3(6,0-9,0)$ & $18,2(16,0-20,7)$ & $16,7((14,6-19,0)$ \\
\hline Sim & $698(37,7)$ & $31,9(28,5-35,5)$ & $9,7(7,7-12,1)$ & $19,6(16,6-22,9)$ & $17,0(14,3-20,1)$ \\
\hline \multicolumn{6}{|l|}{ Compartilha a cama onde dorme } \\
\hline Não & $1.382(74,7)$ & $31,6(29,2-34,1)$ & $8,2(6,9-9,8)$ & $18,4(16,3-20,7)$ & $16,7(14,8-18,8)$ \\
\hline Sim & $469(25,3)$ & $32,4(28,3-36)$, & $8,2(6,0-11,0)$ & $19,7(16,1-23,9)$ & $17,0(13,8-20,8)$ \\
\hline \multicolumn{6}{|l|}{ Aula pela manhã } \\
\hline Não & $793(42,8)$ & $20,2(17,5-23,1)$ & $8,4(6,6-10,5)$ & $19,8(17,0-22,9)$ & $17,4(14,8-20,3)$ \\
\hline Sim & $1.061(57,2)$ & $40,6(37,7-43,6)$ & $8,1(6,7-9,9)$ & $18,0(15,7-20,6)$ & $17,0(14,8-19,4)$ \\
\hline \multicolumn{6}{|l|}{ Uso de tela (horas) } \\
\hline 1ㅇtercil & $749(41,2)$ & $32,2(29,0-35,8)$ & $7,3(5,6-9,4)$ & $17,7(15,0-20,8)$ & $15,6(13,1-18,4)$ \\
\hline 2o tercil & $533(29,3)$ & $32,3(28,5-36,4)$ & $7,5(5,6-10,1)$ & $15,9(12,9-19,5)$ & $16,2(13,3-19,7)$ \\
\hline 3o tercil & $537(29,5)$ & $31,1(27,3-35,2)$ & $9,2(7,0-11,9)$ & $22,4(18,9-26,5)$ & $19,7(16,4-23,4)$ \\
\hline \multicolumn{6}{|l|}{ Atividade física } \\
\hline Ativo & $1.025(55,0)$ & $32,3(29,5-35,2)$ & $9,3(7,7-11,3)$ & $18,6(16,2-21,3)$ & $17,5(15,2-20,0)$ \\
\hline Inativo & $837(45,0)$ & $31,2(28,2-34,4)$ & $6,8(5,2-8,7)$ & $18,6(16,0-21,6)$ & $16,8(14,3-19,5)$ \\
\hline \multicolumn{6}{|l|}{ Fuma } \\
\hline Não & $1.659(89,0)$ & $31,6(29,4-33,9)$ & $7,5(6,3-8,9)$ & $18,3(16,4-20,3)$ & $17,2(15,4-19,1)$ \\
\hline Sim & $204(11,0)$ & $33,5(27,3-40,3)$ & $13,3(9,3-18,7)$ & $21,7(16,2-28,5)$ & $16,9(12,2-23,0)$ \\
\hline \multicolumn{6}{|l|}{ Consumo nocivo de álcool } \\
\hline Não & $1.140(66,7)$ & $29,9(27,3-32,7)$ & $7,3(5,9-9,0)$ & $17,1(14,9-19,6)$ & $16,9(14,8-19,3)$ \\
\hline Sim & $568(33,3)$ & $36,5(32,6-40,5)$ & $9,6(7,4-12,3)$ & $21,5(18,1-25,3)$ & $17,7(14,7-21,2)$ \\
\hline \multicolumn{6}{|l|}{ Área do curso } \\
\hline $\begin{array}{l}\text { Ciências Exatas e da Terra/ } \\
\text { Agrárias e Engenharias }\end{array}$ & $544(29,2)$ & $39,4(35,4-43,6)$ & $8,3(6,3-11,0)$ & $17,1(14,0-20,7)$ & $16,7(13,8-20,2)$ \\
\hline Ciências da Saúde e Biológicas & $332(17,8)$ & $37,1(32,0-42,5)$ & $6,1(3,9-9,2)$ & $17,0(13,1-21,8)$ & $18,5(14,6-23,2)$ \\
\hline $\begin{array}{l}\text { Ciências Sociais Aplicadas e } \\
\text { Humanas }\end{array}$ & $641(34,4)$ & $25,5(22,2-29,0)$ & $6,9(5,2-9,2)$ & $18,7(15,7-22,2)$ & $16,8(14,0-19,9)$ \\
\hline Linguística, Letras e Artes & $348(18,7)$ & $26,2(21,9-31,1)$ & $12,1(9,1-16,0)$ & $22,6(18,2-27,6)$ & $17,3(13,6-21,8)$ \\
\hline
\end{tabular}

\section{Sono insuficiente nos dias de aula}

A duração média do sono dos estudantes nos dias de aula foi de 6,5 horas. Cerca de $32 \%$ dos alunos apresentaram duração de sono insuficiente nos dias de aula, sendo esta mais prevalente entre os indivíduos de cor preta (37,2\%), que estudavam de manhã (40,6\%), com consumo nocivo de álcool (36,5\%) e de cursos da área de Ciências Exatas e da Terra/Agrárias e Engenharias (39,4\%) (Tabela 1). Nas análises ajustadas, a probabilidade de apresentar duração do sono noturno abaixo do recomendado nos dias de aula foi $26 \%$ maior entre os estudantes de cor preta $(\mathrm{RP}=1,26)$, em comparação aos de 
cor branca (Tabela 2). Entre os alunos com aulas no turno da manhã, a probabilidade de duração insuficiente de sono nos dias de aula foi duas vezes maior do que entre os estudantes dos demais turnos $(\mathrm{RP}=2,08)$. O consumo nocivo de álcool esteve associado a uma probabilidade $17 \%$ maior de duração insuficiente do sono nos dias de aula $(\mathrm{RP}=1,17)$ (Tabela 2$)$.

\section{Sono insuficiente nos finais de semana}

A duração média do sono dos estudantes nos finais de semana é de 8,1 horas. A prevalência de duração de sono insuficiente foi de $8,2 \%$, sendo mais frequente entre os homens (9,8\%), indivíduos com mais de 23 anos (11,6\%), fisicamente ativos (9,3\%), fumantes (13,3\%) e de cursos da área de Linguística, Letras e Artes (12,1\%) (Tabela 1). A probabilidade de duração do sono abaixo do recomendado foi cerca de $70 \%$ maior entre os fumantes ( $R P=1,66)$, quando comparados aos não fumantes, e aproximadamente $21 \%$ menor entre os fisicamente ativos $(\mathrm{RP}=0,71)$ comparando-os aos sedentários (Tabela 2).

\section{Tempo de latência longo nos dias de aula}

Em toda a amostra, a mediana e o intervalo interquartil do período de latência do sono foi de 20 minutos (IIQ = 10,0-30,0 minutos) nos dias de aula. A prevalência de latência longa (maior que 30 minutos) nos dias de aula foi maior entre os que faziam uso nocivo de álcool (21,5\%) (Tabela 1). Após ajuste, a latência longa do sono nos dias de aula esteve associada à cor da pele parda $(R P=1,38)$, em comparação aos de cor branca, e ao consumo nocivo de álcool, sendo que entre os estudantes com consumo nocivo a probabilidade de latência longa foi $36 \%$ maior do que entre seus contrapartes $(\mathrm{RP}=1,36)($ Tabela 2$)$.

\section{Tabela 2}

Razões de prevalências (RP) ajustadas para duração insuficiente do sono e latência longa, em dias de aula e em finais de semana, segundo características sociodemográficas e comportamentais de estudantes universitários.

\begin{tabular}{|c|c|c|c|c|}
\hline Características & $\begin{array}{c}\text { Sono noturno } \\
\text { insuficiente nos dias } \\
\text { de aula (IC95\%) }\end{array}$ & $\begin{array}{c}\text { Sono noturno } \\
\text { insuficiente nos finais } \\
\text { de semana (IC95\%) }\end{array}$ & $\begin{array}{c}\text { Latência do sono > } 30 \\
\text { minutos em dias de } \\
\text { aula (IC95\%) }\end{array}$ & $\begin{array}{l}\text { Latência do sono }>30 \\
\text { minutos em finais de } \\
\text { semana (IC95\%) }\end{array}$ \\
\hline \multicolumn{5}{|l|}{ Sexo } \\
\hline Feminino & 1,00 & 1,00 & 1,00 & 1,00 \\
\hline Masculino & $1,06(0,92-1,21)$ & $1,33(0,95-1,85)$ & $0,82(0,65-1,04)$ & $0,86(0,70-1,07)$ \\
\hline \multicolumn{5}{|l|}{ Cor da pele } \\
\hline Branca & 1,00 & 1,00 & 1,00 & * \\
\hline Parda & $1,14(0,94-1,38)$ & $1,18(0,78-1,91)$ & $1,38(1,03-1,85)$ & * \\
\hline Preta & $1,26(1,04-1,52)$ & $1,22(0,78-1,91)$ & $1,14(0,81-1,59)$ & * \\
\hline \multicolumn{5}{|l|}{ Idade (anos) } \\
\hline $18-19$ & 1,00 & 1,00 & * & * \\
\hline $20-22$ & $0,70(0,76-1,06)$ & $0,87(0,57-1,34)$ & * & * \\
\hline$\geq 23$ & $1,13(0,95-1,34)$ & $1,43(0,95-2,15)$ & * & * \\
\hline \multicolumn{5}{|l|}{ Classe social } \\
\hline A & * & 1,00 & 1,00 & * \\
\hline B & * & $1,43(0,81-2,50)$ & $0,84(0,61-1,15)$ & * \\
\hline C & * & $1,52(0,85-2,70)$ & $0,95(0,69-1,32)$ & * \\
\hline D-E & * & $1,04(0,36-3,04)$ & $0,57(0,27-1,22)$ & * \\
\hline \multicolumn{5}{|c|}{ Compartilha a casa onde mora } \\
\hline Não & * & 1,00 & * & * \\
\hline Sim & * & $1,85(0,87-3,96)$ & $\star$ & * \\
\hline
\end{tabular}

(continua) 
Tabela 2 (continuação)

\begin{tabular}{|c|c|c|c|c|}
\hline Características & $\begin{array}{c}\text { Sono noturno } \\
\text { insuficiente nos dias } \\
\text { de aula (IC95\%) }\end{array}$ & $\begin{array}{c}\text { Sono noturno } \\
\text { insuficiente nos finais } \\
\text { de semana (IC95\%) }\end{array}$ & $\begin{array}{l}\text { Latência do sono > } 30 \\
\text { minutos em dias de } \\
\text { aula (IC95\%) }\end{array}$ & $\begin{array}{c}\text { Latência do sono }>30 \\
\text { minutos em finais de } \\
\text { semana (IC95\%) }\end{array}$ \\
\hline \multicolumn{5}{|l|}{$\begin{array}{l}\text { Compartilha o quarto onde } \\
\text { dorme }\end{array}$} \\
\hline Não & * & 1,00 & * & * \\
\hline Sim & * & $1,21(0,86-1,70)$ & * & * \\
\hline \multicolumn{5}{|l|}{$\begin{array}{l}\text { Compartilha a cama onde } \\
\text { dorme }\end{array}$} \\
\hline Não & * & * & * & * \\
\hline Sim & * & * & * & * \\
\hline \multicolumn{5}{|l|}{ Aula pela manhã } \\
\hline Não & 1,00 & * & * & * \\
\hline Sim & $2,08(1,70-2,55)$ & * & * & * \\
\hline \multicolumn{5}{|l|}{ Uso de tela (horas) } \\
\hline 1o tercil & * & * & 1,00 & 1,00 \\
\hline 2o tercil & * & * & $0,86(0,65-1,14)$ & $1,03(0,80-1,34)$ \\
\hline 3o tercil & * & * & $1,12(0,87-1,46)$ & $1,25(0,98-1,60)$ \\
\hline \multicolumn{5}{|l|}{ Atividade física } \\
\hline Ativo & * & 1,00 & * & * \\
\hline Inativo & * & $0,71(0,50-1,00)$ & * & * \\
\hline \multicolumn{5}{|l|}{ Fuma } \\
\hline Não & * & 1,00 & * & * \\
\hline Sim & * & $1,66(1,01-2,53)$ & * & * \\
\hline \multicolumn{5}{|l|}{ Consumo nocivo de álcool } \\
\hline Não & 1,00 & 1,00 & 1,00 & * \\
\hline Sim & $1,17(1,02-1,35)$ & $1,19(0,83-1,71)$ & $1,36(1,09-1,71)$ & * \\
\hline \multicolumn{5}{|l|}{ Área do curso } \\
\hline $\begin{array}{l}\text { Ciências Exatas e da Terra/ } \\
\text { Agrárias e Engenharias }\end{array}$ & 1,00 & 1,00 & 1,00 & * \\
\hline $\begin{array}{l}\text { Ciências da Saúde e } \\
\text { Biológicas }\end{array}$ & $0,94(0,78-1,12)$ & $0,80(0,47-1,36)$ & $0,91(0,64-1,30)$ & * \\
\hline $\begin{array}{l}\text { Ciências Sociais Aplicadas e } \\
\text { Humanas }\end{array}$ & $1,01(0,82-1,24)$ & $0,75(0,48-1,16)$ & $1,05(0,79-1,40)$ & * \\
\hline Linguística, Letras e Artes & $0,89(0,71-1,11)$ & $1,28(0,85-1,92)$ & $1,22(0,89-1,67)$ & * \\
\hline
\end{tabular}

Nota: variáveis mantidas para a análise ajustada (valor de $\mathrm{p}$ da associação com o desfecho $\leq 0,20$ ): Sono noturno < recomendado nos dias de aula (sexo, cor da pele, idade, aula pela manhã, consumo nocivo de álcool e área do curso); Sono noturno < recomendado nos finais de semana (sexo, cor da pele, idade, classe social, compartilha casa onde mora, compartilha o quarto onde dorme, atividade física, fumo, consumo nocivo de álcool e área do curso); latência > 30 minutos nos dias úteis (sexo, cor da pele, classe social, número de horas de tela, consumo nocivo de álcool e área do curso); latência > 30 minutos nos finais de semana (sexo e número de horas de tela).

* Indica que a variável da linha correspondente não foi levada para análise multivariada por não associar-se $(p<0,20)$ ao desfecho.

\section{Tempo de latência longo nos finais de semana}

A mediana e o intervalo interquartil do período de latência do sono foi de 15 minutos (IIQ = 5,0-30,0 minutos) nos finais de semana. A prevalência de latência longa nos finais de semana não esteve associada com nenhuma das variáveis independentes investigadas (Tabela 1).

\section{Sono de baixa qualidade}

A prevalência autopercebida de sono de baixa qualidade foi de $30 \%$, sendo maior entre as mulheres $(32,6 \%)$, indivíduos de cor de pele parda $(36,6 \%)$ ou preta $(34,7 \%)$, fumantes $(38,5 \%)$, com consumo 
nocivo de álcool (35,4\%) e que referiram mais de cinco horas diárias de uso de tela (36,5\%) (Tabela 2). A prevalência de sono de baixa qualidade autopercebida foi menor entre os estudantes que moravam sozinhos (23,3\%) (Tabela 3$)$.

As $\mathrm{RP}$ ajustadas foram maiores entre os indivíduos de cor parda $(\mathrm{RP}=1,38)$ ou preta $(\mathrm{RP}=1,23)$, que compartilhavam a casa $(R P=1,47)$, com mais de cinco horas diárias de uso de tela $(R P=1,24)$, fumantes $(\mathrm{RP}=1,28)$ e com consumo nocivo de álcool $(\mathrm{RP}=1,27)$ (Tabela 4). A probabilidade de sono de baixa qualidade autopercebida foi $16 \%$ menor $(\mathrm{RP}=0,84)$ entre os homens, comparados às mulheres (Tabela 4).

\section{Despertares noturnos}

A prevalência de despertares noturnos na maioria das noites ou sempre foi de $12,7 \%$, sendo mais frequente entre as estudantes de sexo feminino (15,2\%), de cor preta (19,1\%), fumantes (20,9\%) e com consumo nocivo de álcool (35,4\%) (Tabela 3).

Nas análises ajustadas, a probabilidade de despertares noturnos foi $54 \%$ maior entre os alunos de cor preta $(R P=1,54)$, em relação aos de cor branca, e $83 \%$ maior entre os fumantes $(R P=1,83)$, comparados aos não fumantes, e com consumo nocivo de álcool $(\mathrm{RP}=1,49)$. Entre os homens, a probabilidade de despertares noturnos foi $38 \%$ menor do que entre as mulheres $(\mathrm{RP}=0,62)$ (Tabela 4$)$.

\section{Tabela 3}

Prevalências de sono de baixa qualidade, despertares noturnos e sonolência diurna, segundo características sociodemográficas e comportamentais de estudantes universitários ( $n=1.865$ ).

\begin{tabular}{|c|c|c|c|}
\hline Características & $\begin{array}{l}\text { Qualidade do sono } \\
\text { (ruim/péssima) } \\
\text { \% (IC95\%) }\end{array}$ & $\begin{array}{c}\text { Despertares noturnos } \\
\text { (maioria das noites/sempre) } \\
\%(\text { IC95\%) }\end{array}$ & $\begin{array}{c}\text { Sonolência diurna } \\
\text { (maioria dos dias/sempre) } \\
\%(\text { IC95\%) }\end{array}$ \\
\hline Total & $30,1(28,0-32,3)$ & $12,7(11,2-14,3)$ & $32,2(30,1-34,4)$ \\
\hline \multicolumn{4}{|l|}{ Sexo } \\
\hline Masculino & $27,2(24,2-30,3)$ & $9,7(7,9-11,9)$ & $25,2(22,4-28,3)$ \\
\hline Feminino & $32,6(29,8-35,6)$ & $15,2(13,1-17,5)$ & $37,9(35,0-40,9)$ \\
\hline \multicolumn{4}{|l|}{ Cor da pele } \\
\hline Branca & $28,3(25,9-30,8)$ & $11,7(10,0-13,5)$ & $32,1(29,6-34,6)$ \\
\hline Parda & $36,6(30,8-42,8)$ & $12,6(9,0-17,3)$ & $34,0(28,3-40,2)$ \\
\hline Preta & $34,7(29,0-41,0)$ & $19,1(14,6-24,6)$ & $32,8(27,1-39,0)$ \\
\hline \multicolumn{4}{|l|}{ Idade (anos) } \\
\hline 18-19 & $29,2(26,1-32,6)$ & $12,4(10,2-14,9)$ & $36,4(33,1-39,9)$ \\
\hline $20-22$ & $29,5(25,9-33,2)$ & $12,2(9,8-15,0)$ & $30,6(27,1-34,4)$ \\
\hline$\geq 23$ & $32,0(27,9-36,3)$ & $14,1(11,2-17,5)$ & $27,6(23,7-31,8)$ \\
\hline \multicolumn{4}{|l|}{ Classe social } \\
\hline A & $30,5(25,2-36,3)$ & $13,6(9,9-18,3)$ & $29,3(24,1-35,1)$ \\
\hline B & $29,5(26,4-32,8)$ & $12,8(10,6-15,3)$ & $32,8(29,6-36,2)$ \\
\hline C & $30,6(27,1-34,3)$ & $12,6(10,3-15,4)$ & $31,6(28,1-35,3)$ \\
\hline$D-E$ & $35,1(25,1-46,5)$ & $11,7(6,1-21,2)$ & $36,4(26,3-47,8)$ \\
\hline \multicolumn{4}{|c|}{ Compartilha a casa onde mora } \\
\hline Não & $23,3(17,7-30,1)$ & $10,0(6,4-15,4)$ & $30,0(23,7-37,1)$ \\
\hline Sim & $30,9(28,7-33,1)$ & $13,0(11,5-14,7)$ & $32,4(30,2-34,6)$ \\
\hline \multicolumn{4}{|c|}{ Compartilha o quarto onde dorme } \\
\hline Não & $30,2(27,7-33,0)$ & $12,1(10,3-14,1)$ & $32,8(30,2-45,6)$ \\
\hline Sim & $29,9(26,7-33,5)$ & $13,8(11,4-16,6)$ & $31,2(27,9-34,8)$ \\
\hline
\end{tabular}

(continua) 
Tabela 3 (continuação)

\begin{tabular}{|c|c|c|c|}
\hline Características & $\begin{array}{l}\text { Qualidade do sono } \\
\text { (ruim/péssima) } \\
\text { \% (IC95\%) }\end{array}$ & $\begin{array}{c}\text { Despertares noturnos } \\
\text { (maioria das noites/sempre) } \\
\%(\mathrm{IC} 95 \%)\end{array}$ & $\begin{array}{c}\text { Sonolência diurna } \\
\text { (maioria dos dias/ sempre) } \\
\%(\text { IC95\%) }\end{array}$ \\
\hline \multicolumn{4}{|l|}{ Compartilha a cama onde dorme } \\
\hline Não & $36,6(28,2-33,0)$ & $12,0(10,4-13,8)$ & $31,9(29,5-34,4)$ \\
\hline Sim & $29,0(25,1-33,3)$ & $14,8(11,9-18,3)$ & $33,3(29,1-37,7)$ \\
\hline \multicolumn{4}{|l|}{ Aula pela manhã } \\
\hline Não & $29,9(26,8-33,2)$ & $13,5(11,3-16,1)$ & $27,3(24,3-30,5)$ \\
\hline $\operatorname{Sim}$ & $30,3(27,6-33,1)$ & $12,2(10,4-14,3)$ & $36,1(33,2-39,0)$ \\
\hline \multicolumn{4}{|l|}{ Uso de tela (horas) } \\
\hline 1o tercil & $27,9(24,7-31,2)$ & $11,4(9,3-13,8)$ & $30,3(27,1-33,7)$ \\
\hline 2o tercil & $26,4(22,8-30,3)$ & $11,9(9,4-14,9)$ & $27,4(23,8-31,4)$ \\
\hline 3o tercil & $36,5(32,5-40,6)$ & $14,8(12,0-18,0)$ & $37,4(33,4-41,6)$ \\
\hline \multicolumn{4}{|l|}{ Atividade física } \\
\hline Ativo & $29,1(26,3-31,2)$ & $12,3(10,4-14,4)$ & $29,3(26,6-32,2)$ \\
\hline Inativo & $31,5(28,4-34,7)$ & $13,3(11,1-15,7)$ & $35,8(32,6-39,1)$ \\
\hline \multicolumn{4}{|l|}{ Fuma } \\
\hline Não & $29,1(27,0-31,4)$ & $11,7(10,3-13,4)$ & $31,6(29,4-33,9)$ \\
\hline Sim & $38,5(32,0-45,5)$ & $20,9(15,8-27,1)$ & $37,3(30,9-44,2)$ \\
\hline \multicolumn{4}{|l|}{ Consumo nocivo de álcool } \\
\hline Não & $27,4(24,8-30,0)$ & $10,8(9,1-12,7)$ & $28,7(26,1-31,4)$ \\
\hline Sim & $35,4(31,5-39,4)$ & $17,0(14,2-20,4)$ & $39,6(35,6-43,7)$ \\
\hline \multicolumn{4}{|l|}{ Área do curso } \\
\hline $\begin{array}{l}\text { Ciências Exatas e da Terra/Agrárias e } \\
\text { Engenharias }\end{array}$ & $27,8(24,2-31,8)$ & $10,4(8,1-13,3)$ & $31,1(27,3-35,2)$ \\
\hline Ciências da Saúde e Biológicas & $29,7(25,0-34,9)$ & $12,7(9,5-16,8)$ & $40,9(35,7-46,3)$ \\
\hline Ciências Sociais Aplicadas e Humanas & $32,7(29,1-36,4)$ & $13,9(11,4-16,8)$ & $32,1(28,6-35,8)$ \\
\hline Linguística, Letras e Artes & $29,5(24,9-34,5)$ & $14,2(10,9-18,3)$ & $26,0(21,6-30,9)$ \\
\hline
\end{tabular}

\section{Tabela 4}

Razões de prevalências (RP) ajustadas para baixa qualidade autopercebida do sono, despertares noturnos e sonolência diurna, segundo características sociodemográficas e comportamentais de estudantes universitários.

\begin{tabular}{lccc}
\hline Características & $\begin{array}{c}\text { Qualidade do sono } \\
\text { (ruim/péssima) } \\
\text { (IC95\%) }\end{array}$ & $\begin{array}{c}\text { Despertares noturnos } \\
\text { (maioria das noites/sempre) } \\
\text { (IC95\%) }\end{array}$ & $\begin{array}{c}\text { Sonolência diurna } \\
\text { (maioria dos dias/sempre) } \\
\text { (IC95\%) }\end{array}$ \\
\hline $\begin{array}{l}\text { Sexo } \\
\text { Feminino }\end{array}$ & 1,00 & 1,00 & 1,00 \\
$\quad \begin{array}{l}\text { Masculino } \\
\text { Cor da pele }\end{array}$ & $0,84(0,73-0,98)$ & $0,62(0,47-0,81)$ & $0,65(0,56-0,76)$ \\
Branca & 1,00 & 1,00 & $*$ \\
Parda & $1,38(1,14-1,67)$ & $1,02(0,69-1,49)$ & $*$ \\
Preta & $1,23(1,00-1,51)$ & $1,54(1,12-2,11)$ & $*$ \\
Idade (anos) & $*$ & $*$ & 1,00 \\
18-19 & $*$ & $*$ & $0,90(0,76-1,06)$ \\
$20-22$ & $*$ & $*$ & $0,94(0,78-1,13)$ \\
$\geq 23$ & & $*$ & $*$
\end{tabular}

(continua) 
Tabela 4 (continuação)

\begin{tabular}{|c|c|c|c|}
\hline Características & $\begin{array}{l}\text { Qualidade do sono } \\
\text { (ruim/péssima) } \\
\text { (IC95\%) }\end{array}$ & $\begin{array}{c}\text { Despertares noturnos } \\
\text { (maioria das noites/sempre) } \\
\text { (IC95\%) }\end{array}$ & $\begin{array}{c}\text { Sonolência diurna } \\
\text { (maioria dos dias/sempre) } \\
\text { (IC95\%) }\end{array}$ \\
\hline \multicolumn{4}{|l|}{ Classe social } \\
\hline A & * & * & * \\
\hline B & * & * & * \\
\hline$c$ & * & * & * \\
\hline D-E & * & * & * \\
\hline \multicolumn{4}{|l|}{ Compartilha a casa onde mora } \\
\hline Não & 1,00 & * & * \\
\hline $\operatorname{Sim}$ & $1,47(1,08-2,00)$ & * & * \\
\hline \multicolumn{4}{|l|}{ Compartilha o quarto onde dorme } \\
\hline Não & * & * & * \\
\hline Sim & * & * & * \\
\hline \multicolumn{4}{|l|}{ Compartilha a cama onde dorme } \\
\hline Não & * & 1,00 & * \\
\hline Sim & * & $1,11(0,84-1,47)$ & * \\
\hline \multicolumn{4}{|l|}{ Aula pela manhã } \\
\hline Não & * & * & 1,00 \\
\hline $\operatorname{Sim}$ & * & * & $1,45(1,20-1,77)$ \\
\hline \multicolumn{4}{|l|}{ Uso de tela (horas) } \\
\hline 1o tercil & 1,00 & 1,00 & 1,00 \\
\hline 2o tercil & $0,93(0,77-1,12)$ & $1,04(0,76-1,44)$ & $0,87(0,73-1,04)$ \\
\hline 3o tercil & $1,24(1,04-1,47)$ & $1,24(0,92-1,67)$ & $1,17(1,00-1,38)$ \\
\hline \multicolumn{4}{|l|}{ Atividade física } \\
\hline Ativo & * & * & 1,00 \\
\hline Inativo & * & * & $1,13(0,98-1,29)$ \\
\hline \multicolumn{4}{|l|}{ Fuma } \\
\hline Não & 1,00 & 1,00 & 1,00 \\
\hline Sim & $1,28(1,05-1,56)$ & $1,83(1,34-2,51)$ & $1,20(0,98-1,47)$ \\
\hline \multicolumn{4}{|l|}{ Consumo nocivo de álcool } \\
\hline Não & 1,00 & 1,00 & 1,00 \\
\hline $\operatorname{Sim}$ & $1,27(1,09-1,48)$ & $1,49(1,15-1,93)$ & $1,30(1,13-1,51)$ \\
\hline \multicolumn{4}{|l|}{ Área do curso } \\
\hline \multicolumn{4}{|l|}{ Engenharias } \\
\hline Ciências da Saúde e Biológicas & $1,10(0,88-1,38)$ & $1,11(0,74-1,67)$ & $1,29(1,07-1,56)$ \\
\hline Ciências Sociais Aplicadas e Humanas & $1,10(0,91-1,33)$ & $1,18(0,85-1,66)$ & $1,29(1,03-1,60)$ \\
\hline Linguística, Letras e Artes & $1,03(0,82-1,29)$ & $1,20(0,81-1,77)$ & $0,95(0,73-1,22)$ \\
\hline
\end{tabular}

Nota: variáveis mantidas para a análise ajustada (valor $p$ da associação com o desfecho $\leq 0,20$ ): Qualidade do sono (sexo, cor da pele, compartilha a casa onde mora, número de horas de tela, fumo, consumo nocivo de álcool e área do curso); Despertares noturnos (sexo, cor da pele, compartilha a cama onde dorme, número de horas de tela, fumo, consumo nocivo de álcool e área do curso); Sonolência diurna (sexo, idade, aula pela manhã, número horas de tela, atividade física, fumo, consumo nocivo de álcool e área do curso).

* Indica que a variável da linha correspondente não foi levada para análise multivariada por não associar-se $(p<0,20)$ ao desfecho. 


\section{Sonolência diurna}

A sonolência diurna foi reportada por $32,2 \%$ dos estudantes, sendo sua frequência mais elevada no sexo feminino (37,9), entre os mais jovens (18-19 anos) (36,4\%), com aulas no turno da manhã $(36,1 \%)$, com mais de cinco horas diárias de uso de tela (37,4\%), que não praticavam atividade física (35,8\%), que relataram consumo nocivo de álcool $(39,6 \%)$ e matriculados em cursos da área de Ciências da Saúde e Biológicas (40,9\%) (Tabela 3).

A RP ajustada foi $37 \%$ maior entre os alunos com aulas no turno da manhã ( $R P=1,37), 32 \%$ maior entre aqueles com consumo nocivo de álcool $(R P=1,32), 29 \%$ maior entre os da área de Ciências da Saúde e Biológicas $(R P=1,29)$ e de Ciências Sociais Aplicadas e Humanas ( $R P=1,29)$, em comparação aos seus controles. A probabilidade de sonolência diurna foi $35 \%$ menor entre os homens $(\mathrm{RP}=0,53)$, quando comparados às mulheres (Tabela 4).

\section{Discussão}

Neste estudo, foram encontradas as seguintes prevalências: sono insuficiente nos dias de aula, 32\%; sono insuficiente nos finais de semana, 8,2\%; latência longa nos dias de aula, 18,6\%; latência longa nos finais de semana, 17,2\%; baixa qualidade autopercebida, 30\%; despertares noturnos, 12,7\%; e sonolência diurna, 32,2\%. O maior consumo de álcool associou-se ao maior número de distúrbios (duração insuficiente e latência longa nos dias de aula, baixa qualidade, despertares e sonolência), seguido do tabagismo (duração insuficiente nos finais de semana, despertares e baixa qualidade) e cor da pele preta ou parda (duração insuficiente nos dias de aula, baixa qualidade e despertares). Aulas no turno da manhã foram associadas com sono insuficiente nos dias de aula e sonolência diurna; e o sexo feminino, com sonolência, baixa qualidade e despertares.

A prevalência de sono insuficiente nos dias de aula (32\%) foi $40 \%$ menor do que a estimada no planejamento deste estudo (53\%) 9 . Já nos finais de semana, a prevalência de sono insuficiente $(8,2 \%)$ foi $84 \%$ menor do que a prevista 9 . Essa diferença pode ser atribuída à idade dos participantes, já que no estudo de Roberts \& Duong 9 foram avaliados indivíduos entre 11 e 17 anos. Os adolescentes podem ter menos horas de sono, em comparação aos de maior idade abordados em nosso estudo. Na adolescência, identificou-se maior lentidão na inibição da secreção de melatonina no início da fase clara do dia, especialmente nas etapas tardias da puberdade, o que pode resultar em diminuição das horas de sono e aumento da sonolência diurna excessiva 19 .

A prevalência de tempo de latência longo, cuja estimativa foi de $65 \% 15$, foi cerca de 3,6 vezes menor em nosso estudo, tendo sido relatada por 18,6\% dos estudantes ao referirem-se aos dias de aula e $17,2 \%$ aos finais de semana. As amostras dos dois estudos foram heterogêneas em relação à faixa etária, sendo a média de idade de 17 anos no trabalho usado como referência e de aproximadamente 22 anos em nosso trabalho. Além disso, apesar de Hysing et al. 15 também terem utilizado um questionário autoaplicado, o respondente pode ter interpretado o tempo em que ficou na cama lendo ou utilizando algum dispositivo eletrônico como período de latência. O questionário aplicado em nosso estudo, por conter ilustrações sobre as etapas do sono, pode ter evitado esse equívoco, reduzindo a prevalência de latência longa do sono 15 .

Cerca de um terço dos estudantes reportaram sono de baixa qualidade. Um estudo brasileiro mostrou que, entre 2001 e 2011, houve um aumento de 26,3\% para 34,5\% na frequência de sono de má qualidade autorreferida e de $37 \%$ para $54 \%$ de sono insuficiente 10 . Entre alunos de medicina de uma universidade pública do Nordeste, a prevalência de sono de má qualidade autorreferida foi de $50 \% 20$.

Ainda, a prevalência de despertares noturnos (12,7\%) foi praticamente a metade da estimada (25\%) 21. Essa discrepância pode ser explicada pelo fato de aqueles autores terem avaliado o sono de pré-escolares. Um estudo realizado com adultos de 20 anos ou mais, em São Paulo, encontrou prevalência relatada de insônia, que incluía latência maior que 30 minutos, despertares noturnos e/ ou despertares precoces (cedo de manhã), nos últimos 30 dias ou mais, com prejuízo das atividades diárias, de 15\% (12,0-18,5) 22. Essas amplas diferenças afetaram o poder do estudo em detectar possíveis associações com as exposições investigadas. Mantidas as razões de prevalências observadas, para duração insuficiente do sono em dias de aula, por exemplo, havia poder de $80 \%$ para detectar 
associação somente com o turno das aulas; para sono insuficiente nos finais de semana, somente com tabagismo; para despertares noturnos, com sexo, cor, tabagismo e consumo nocivo de álcool; e, para latência longa, o poder para todas as exposições era inferior a 80\%.

As prevalências de sono insuficiente nos dias de aula e sonolência diurna foram muito semelhantes (em torno de 32\%). Dentre os que apresentavam sonolência, 40,6\% reportavam também sono insuficiente nos dias de aula, sendo a prevalência de sonolência $40 \%$ maior entre os estudantes com sono insuficiente, mesmo após ajuste para sexo, índice de bens, compartilhamento do quarto e da cama, número de horas diárias de tela, aulas no turno da manhã e consumo nocivo de álcool. Vários estudos têm apontado o horário de início das aulas como um fator associado a distúrbios do sono entre escolares 23,24 .

Cerca de um terço dos alunos apresentava sono insuficiente nos dias de aula, sendo a probabilidade deste desfecho duas vezes maior entre os que assistiam às aulas no turno da manhã, em comparação aos que tinham aulas nos demais turnos. O horário das aulas pode desregular o padrão do sono, pois os estudantes sincronizam o ciclo sono-vigília diferentemente do ciclo claro-escuro (alternância do dia-noite), para tentar cumprir suas atividades 5,25. Menos de 10\% dos alunos apresentaram duração do sono inferior ao recomendado nos finais de semana, sugerindo que dormem menos nos dias de aula e compensam o déficit nos dias livres.

Consistente com as prevalências de duração insuficiente do sono e sonolência diurna, foi a autoavaliação de sono de baixa qualidade (ruim ou muito ruim) relatada por cerca de um terço dos estudantes. Tal achado é semelhante ao encontrado por Hoefelmann et al. 10, entre escolares do Estado de Santa Catarina 10. Várias características dos estudantes associaram-se à baixa qualidade autopercebida, sendo o compartilhamento da casa o aspecto mais fortemente associado, aumentando em cerca de 50\% a probabilidade do relato, em comparação aos que moravam sozinhos. Seguiram-se estudantes do sexo feminino, de cor parda ou preta, com alto uso de telas, fumantes e com consumo nocivo de álcool. O número de pessoas no quarto e na cama pode influenciar negativamente o sono, por ronco do parceiro e limitação do espaço para movimentação no leito 26. Contudo, em nosso trabalho, não foram encontradas essas associações. Estudos mostraram que o uso de computadores e outros equipamentos eletrônicos, diariamente e por longos períodos de tempo, tem como consequência o aumento do tempo de latência e redução da duração do sono 27 . O acesso à tecnologia e o uso "abusivo" de aparelhos eletrônicos têm diminuído tanto o número de horas quanto a qualidade do sono de jovens estudantes 28 . Além disso, outros estímulos como os dispositivos eletrônicos (smart phones, videogames, televisão e tablets), que constituem elemento importante da vida social, podem aumentar o tempo de latência, reduzindo assim a duração do sono noturno 27. Como em nosso estudo, Lil et al. 29 também identificaram que o tabagismo estava associado à má qualidade do sono em adolescentes e adultos, resultando em sono mais superficial, agitado e com despertares 29 . Assim como o tabagismo, outro estudo também mostrou que o consumo de bebidas alcoólicas estava associado com a maior prevalência de insônia e má qualidade do sono 30 .

Dentre todas as características avaliadas, o consumo nocivo de álcool foi a que se associou com o maior número de distúrbios (duração insuficiente e latência longa nos dias de aula, baixa qualidade autopercebida, despertares noturnos e sonolência diurna). Os efeitos nocivos do álcool sobre a saúde são bem conhecidos 31 e sua relação com problemas de sono foi bem documentada em estudos com adultos 32 e adolescentes 33 . O consumo frequente ou a dependência pode aumentar o número de despertares e a latência, e reduzir o tempo total de sono 34 . Vale ressaltar a frequência expressiva de estudantes que faziam consumo nocivo de bebidas alcoólicas nesta fase de início da vida acadêmica $(33,3 \%)$, em razão dos riscos que este comportamento acarreta para a saúde do indivíduo.

Depois do consumo nocivo de álcool, as características associadas com o maior número de distúrbios foram o tabagismo e a cor preta ou parda. Estudantes fumantes apresentaram maiores prevalências de duração insuficiente nos finais de semana, despertares noturnos e baixa qualidade autopercebida. Consistente com esse resultado, um inquérito de base populacional nos Estados Unidos mostrou que os fumantes apresentavam uma prevalência $73 \%$ maior de distúrbios do sono do que os não fumantes 35 . Estudos sugerem que o fumo altera a fisiologia da musculatura respiratória, levando a um quadro de apneia durante o sono, prejudicando sua duração e qualidade 36 . A nicotina, ainda, tem ação estimulante no sistema nervoso central, aumentando o tempo de latência do sono 36 . 
A cor preta ou parda foi associada à duração insuficiente de sono nos dias de aula, sono de baixa qualidade autopercebida e despertares noturnos. A literatura descreve não haver diferença entre o perfil do sono e a cor da pele ${ }^{37}$. Porém, como em nosso estudo, pesquisas realizadas nos Estados Unidos encontraram longo tempo de latência 38 e duração insuficiente 39 entre indivíduos afrodescendentes. Esses indivíduos parecem possuir perfil sociodemográfico e comportamental diferente, como nível social mais baixo, trabalho noturno, compartilhamento da casa onde mora com um número elevado de pessoas, o que poderia contribuir para um sono de baixa duração e qualidade 40 .

O sexo feminino associou-se à sonolência diurna, sono de baixa qualidade autopercebida e despertares noturnos. Alguns estudos não encontraram associação entre sexo e sono 4 , em outros, os distúrbios do sono foram mais prevalentes no sexo masculino do que no feminino 41, e em outros, houve maior prevalência de sono insuficiente, latência longa e sonolência diurna entre as mulheres 15,38.

O grupo econômico da família e o compartilhamento do quarto ou da cama não se associaram com nenhum dos desfechos de sono, tanto nas análises brutas quanto nas ajustadas, o que pode ter ocorrido devido à perda de poder do estudo. E, a atividade física que esteve associada à duração insuficiente do sono nos finais de semana e à sonolência diurna, perdeu a significância estatística após ajuste para covariáveis, o que talvez se deva ao baixo poder do estudo. No entanto, embora haja evidências de que a atividade física possa ser útil no manejo de problemas de sono, ainda não está claro o tipo, duração, intensidade e horário em que esta atividade deva ser realizada de forma a beneficiar o sono na população em geral ${ }^{3}$.

Entre as limitações deste estudo estão a possibilidade de causalidade reversa e confusão residual, uma vez que outros fatores potencialmente associados a distúrbios do sono de estudantes, como estar inserido no mercado de trabalho ${ }^{42}$, consumo de cafeína 43 , estresse 44 e saúde mental 26 não foram investigados. A capacidade de generalização dos achados é afetada pelo fato de nossa amostra ser formada por calouros de uma única universidade, de caráter público e localizada no Sul do país.

A principal contribuição deste estudo é ter medido prevalências de distúrbios do sono em um grupo de estudantes universitários brasileiros, as quais poderão ser úteis para o monitoramento destes agravos ao longo do tempo, na mesma população ou em futuros calouros. Além disso, fornece estimativas de prevalência, muitas das quais, como bem mostramos, diferem das relatadas em estudos internacionais, como, aliás, esperado para desfechos que dependem não apenas de componentes biológicos, mas também de fatores contextuais e culturais, como é o caso dos distúrbios do sono.

\section{Conclusão}

Nosso trabalho mostrou que as aulas no turno da manhã, o sexo feminino e a cor da pele preta, bem como fatores comportamentais (consumo nocivo de bebidas alcoólicas e tabagismo) associaram-se à maioria dos distúrbios do sono investigados. Tanto o consumo de álcool quanto o tabagismo são estilos de vida modificáveis e seu afastamento poderia contribuir para uma adequada higiene do sono entre estudantes universitários. Da mesma forma, ajustes nos horários de início das aulas no turno da manhã, como realizados em outros países 45 , poderiam contribuir na prevenção da má qualidade do sono dos alunos. 


\section{Colaboradores}

C. M. M. Carone e I. S. Santos contribuíram na revisão da literatura, análise e interpretação dos dados, pela redação do artigo e aprovação final da versão a ser publicada. B. D. P. Silva supervisionou a revisão da literatura, bem como análise e interpretação dos dados, orientou a redação do artigo e revisou a versão final a ser publicada. L. T. Rodrigues, P. S. Tavares e M. X. Carpena participaram da análise e interpretação dos dados; auxiliou na revisão crítica relevante do conteúdo intelectual; participou da aprovação final da versão a ser publicada.

\section{Informações adicionais}

ORCID: Caroline Maria de Mello Carone (00000002-8125-586X); Bianca Del Ponte da Silva (0000-0003-2318-6170); Luciana Tovo Rodrigues (0000-0002-8732-6059); Patrice de Souza Tavares (0000-0002-0147-3112); Marina Xavier Carpena (0000-0002-4690-5791); Iná S. Santos (0000-00031258-9249).

\section{Agradecimentos}

Agradecemos à Coordenação de Aperfeiçoamento de Pessoal de Nível Superior (Capes) e ao Conselho Nacional de Desenvolvimento Científico e Tecnológico $(\mathrm{CNPq})$ pelo financiamento deste estudo.

\section{Referências}

1. Shukla C, Basheer R. Metabolic signals in sleep regulation: recent insights. Nat Sci Sleep 2016; 8:9-20.

2. Sadeh A, Tikotzky L, Kahn M. Sleep in infancy and childhood: implications for emotional and behavioral difficulties in adolescence and beyond. Curr Opin Psychiatry 2014; 27:453-9.

3. Irish LA, Kline CE, Gunn HE, Buysse DJ, Hall $\mathrm{MH}$. The role of sleep hygiene in promoting public health: a review of empirical evidence. Sleep Med Rev 2015; 22:23-36.

4. Battacharrya N. Abnormal sleep duration is associated with a higher risk of accidental injury. Otolaryngol Head Neck Surg 2015; 153:962-5.

5. Pereira EG, Gordia AP, Quadros TMB. Padrão do sono em universitários brasileiros e sua relação com a prática de atividades físicas: uma revisão da literatura. Rev Bras Ciênc Saúde 2011; 30:55-60.

6. Fattinger S, Beukelaar TT, Ruddy KL, Volk C, Heyse NC, Herbst JA, et al. Deep sleep maintains learning efficiency of the human brain. Nat Commun 2017; 8:15405.

7. Murillo R, Lambiase MJ, Rockette-Wagner BJ, Kriska AM, Haibach JP, Thurston RC. Racial/ ethnic differences in the associations between physical activity and sleep duration: a population-based study. J Phys Act Health 2017; 14:138-44.

8. Roberts RE, Roberts CR, Xing Y. Restricted sleep among adolescents: prevalence, incidence, persistence, and associated factors. Behav Sleep Med 2011; 9:18-30.

9. Roberts RE, Duong HT. Is there an association between adolescent sleep restriction and obesity. J Psychosom Res 2015; 79:651-6.

10. Hoefelmann LP, Lopes AS, Silva KS, Moritz P, Nahas MV. Sociodemographic factors associated with sleep quality and sleep duration in adolescents from Santa Catarina, Brazil: what changed between 2001 and 2011? Sleep Med 2013; 14:1017-23.

11. Harris PA, Taylor R, Thielke R, Payne J, Gonzales N, Conde JG. Research Eletronic Data Capture (REDCap). A metadata-driven methodology and workflow process for providing translational research informatics support. J Biomed Inform 2009; 42:377-81.

12. Pilz LK, Levandovski R, Oliveira MAB, Hidalgo MP, Roenneberg T. Sleep and light exposure across differente levels of urbanisation in Brazilian communities. Sci Rep 2018; 8:11389.

13. Roenneberg T, Wirz-Justice A, Merrow M. Life between clocks: daily temporal patterns of human chronotypes. J Biol Rhythms 2003; 1:80-90.

14. Hirschkowitz M, Whiton K, Albert SA, Alessi C, Bruni O, Don-Carlos L. The National Sleep Foundation's sleep time duration recommendations: methodology and results summary. Sleep Health 2015; 1:40-3. 
15. Hysing M, Pallesen S, Stormark KM, Jakobsen R, Lundervold AJ, Silvertsen B. Sleep patterns and insomnia among adolescentes: a population-based study. J Sleep Res 2013; 22:549-56.

16. Matsudo S, Araújo T, Natsudo V, Andrade D, Andrade E, Oliveira LC, et al. Questionário Internacional de Atividade Física (IPAQ): estudo de validade e reprodutibilidade no Brasil. Rev Bras Ativ Fís Saúde 2001; 6:5-18.

17. Mendez EB. Uma versão brasileira do AUDIT (Alcohol use Disorders Identification Test). Pelotas: Universidade Federal de Pelotas; 1999.

18. Barbor TF, Higgins-Biddle JC, Monteiro MG. The Alcohol Use Disorders Identification Test - guideline for use in primary care. Geneva: World Health Organization; 2001.

19. Bartel KA, Gradisar M, Williamson P. Protective and risk factors adolescente sleep: a metaanalytic review. Sleep Med Rev 2015; 21:7285.

20. Machado L, Souza CTN, Nunes RO, Santana $\mathrm{CN}$, Araújo CF, Cantilino A. Subjective wellbeing, religiosity and anxiety: a cross-sectional study applied to a sample of Brazilian medical students. Trends Psychiatry Psychoter 2018; 40:185-92.

21. Ivanenko A, Gururaj BR. Classification and epidemiology of sleep disorders. Child Adolesc Psychiatr Clin N Am 2009; 18:839-48.

22. Castro LS, Poyares D, Leger D, Bittencourt L, Tufik S. Objective prevalence of insomnia in the São Paulo, Brazil epidemiologic sleep study. Ann Neurol 2013; 74:537-46.

23. Owens J, Drobnich D, Baylor A, Lewin D. School start time change: an in-depth examination of school districts in the United States. Mind, Brain and Education 2014; 8:182-213.

24. Enright T, Refinetti R. Chronotype, class time, and academic achievement of university students. Chronobiol Int 2017; 34:445-50.

25. Zimmermann LK. Chronotype and the transition to college life. Chronobiol Int 2011; 28:904-10.

26. Sariarslan HA, Gullhan YB, Enalan D, Basturk M, Delibas S. The relationship of sleep problems to life quality and depresssion. Neurosciences 2015; 20:236-42.

27. Hysing M, Pallesen S, Stormark KM, Jakobsen R, Lundervold AJ, Silvertsen B. Sleep and use of electronic devices in adolescence: results from a large population-based study. BMJ Open 2015; 5:e006748.

28. Reid Chassiakos YL, Radesky J, Christakis D, Moreno MA, Cross C; Council on Communications and Media. Children and adolescents and digital media. Pediatrics 2016; 138:e20162593.

29. Lil L, Gong S, Xu C, Zhou JY, Wang KS. Sleep duration and smoking are associated with coronary heart disease among US adults with type 2 diabetes: gender differences. Diabetes Res Clin Pract 2017; 124:93-101.

30. Chen H, Bo QG, Jia CX, Liu X. Sleep problems in relation to smoking and alcohol use in Chinese adolescents. J Nerv Ment Dis 2017; 205:353-60.
31. Reen EV, Tarokh L, Rupp TL, Seifer R, Carskadon MA. Does timing of alcohol administration affect sleep? Sleep 2011; 34:195-205.

32. Morioka $\mathrm{H}$, Itani $\mathrm{O}$, Kaneita $\mathrm{Y}$, Ikeda $\mathrm{M}$, Kondo S, Yamamoto R, et al. Associations between sleep disturbance and alcohol drinking: a large-scale epidemiological study of adolescentes in Japan. Alcohol 2013; 47:619-28.

33. Silvertsen B, Glozier N, Harvey AG, Hysing M. Academic performance in adolescentes with delayed sleep phase. Sleep Med 2015; 16:108490.

34. Garcia AN, Salloum IM. Polysomnographic sleep disturbances in nicotine, caffeine, alcohol, cocaine, opioid, and cannabis use: a focused review. Am J Addict 2015; 24:590-8.

35. Davila EP, Lee DJ, Fleming LE, LeBlanc WG, Arheart K, Dietz N, et al. Sleep disorders and secondhand smoke exposure in the U.S. population. Nicotine Tob Res 2010; 12:294-9.

36. McNamara JPH, Wang J, Holiday DB, Warren JY, Paradoa M, Balkhi AM, et al. Sleep disturbances associated with cigarette smoking. Psychol Health Med 2014; 19:410-9.

37. Whinnery J, Jackson N, Rattanaumpawan P, Grandner MA. Short and long sleep duration associated with race/ethnicity, sociodemographics, and socioeconomic position. Sleep 2014; 37:601-11.

38. Thomas SJ, Lichstein KL, Taylor DJ, Riedel BW, Bush AJ. Epidemiology of bedtime, arising time, and time in bed: analysis of age, gender, and ethnicity. Behav Sleep Med 2014; 12:169-82.

39. Cunningham TJ, Wheaton AG, Ford ES, Croft JB. Racial/ethnic disparities in self-reported short sleep duration among US-born and foreign-born adults. Ethn Health 2016; 21:628-38.

40. Johnson DA, Jackson CL, Williams NJ, Alcántara C. Are sleep patterns influenced by race/ ethnicity - a marker of relative advantage or disadvantage? Evidence to date. Nat Sci Sleep 2019; 11:79-95

41. Merdad RA, Merdad LA, Nassif RA, El-Derwi D, Wali SO. Sleep habits in adolescents of Saudi Arabia: district patterns and extreme sleep schedules. Sleep Med 2014; 15:1370-8.

42. Pereira EF, Bernardo MPSL, D'Almeida V, Louzada FM. Sono, trabalho e estudo: duração do sono em estudantes trabalhadores e não trabalhadores. Cad Saúde Pública 2011; 27:975-84.

43. Drake C, Roehrs T, Shambroom J, Roth T. Caffeine effects on sleep taken 0, 3, or 6 hours before going to bed. J Clin Sleep Med 2013; 9:1195-200.

44. Kim EJ, Dimsdale JE. The effect of psychosocial stress on sleep: a review of polysomnographic evidence. Behav Sleep Med 2007; 5:256-78.

45. Wheaton AG, Chapman DP, Croft JB. School start times, sleep, behavioral, health, and academic outcomes: a review of the literature. J Sch Health 2016; 86:363-81. 


\section{Abstract}

The objective was to investigate sleep disorders and associated sociodemographic and behavioral factors. A census of university students was carried out. Questions extracted from the Munich Chronotype Questionnaire investigated: insufficient sleep duration ( $<6$ hours/day for $<65$ years and $<5$ hours/day for other ages), long latency (> 30 minutes), low self-rated sleep quality, nocturnal awakenings (involuntary, in the middle of the night), and daytime sleepiness (difficulty concentrating). Independent variables included sociodemographic and behavioral characteristics. Adjusted analyses were performed with Poisson regression. Of the 1,865 students, 32\% exhibited insufficient sleep on class days, $8.2 \%$ insufficient sleep on weekends, $18.6 \%$ long latency on class days, $17.2 \%$ long latency on weekends, $30 \%$ low self-rated sleep quality, 12.7\% nocturnal awakenings, and $32.2 \%$ daytime sleepiness. Higher alcohol consumption was associated with insufficient sleep duration and long latency on class days, low quality sleep, awakenings, and daytime sleepiness. Smoking was associated with insufficient sleep duration on weekends, awakenings, and lowquality sleep. Black and brown skin color were associated with insufficient sleep duration on class days, low-quality sleep, and awakenings. Morning classes were associated with insufficient sleep and daytime sleepiness. Female gender was associated with low-quality sleep and awakenings. The most frequent sleep disorders were insufficient sleep on class days, low self-rated quality of sleep, and daytime sleepiness. Alcohol consumption and smoking and black and brown skin color were the principal factors associated with sleep disorders.

Sleep Disorder; Sleep; Sleepness; Students

\section{Resumen}

El objetivo fue investigar trastornos del sueño, factores sociodemográficos y de comportamiento asociados. Fue realizado un censo con los estudiantes universitarios. Las preguntas extraídas del Munich Chonotype Questionnaire investigaron: duración insuficiente del sueño ( $<6$ horas/día para $<65$ años $y<5$ horas/día para los demás), latencia prolongada (> 30 minutos), baja calidad autopercibida, vigilias (involuntarias, en medio de la noche) y somnolencia diurna (dificultad de concentración). Las variables independientes englobaron características sociodemográficas y de comportamiento. Se realizaron análisis ajustados con regresión de Poisson. De los 1.865 estudiantes, un $32 \%$ presentaron sueño insuficiente durante los días de clase, un $8,2 \%$ sueño insuficiente los fines de semana, un 18,6\% latencia prolongada durante los días de clase, un 17,2\% latencia prolongada durante los fines de semana, un 30\% baja calidad autopercibida, un $12,7 \%$ vigilias y un $32,2 \%$ somnolencia diurna. El mayor consumo de alcohol se asoció a la duración insuficiente y latencia prolongada los días de clase, baja calidad de sueño, vigilias y somnolencia; el tabaquismo con duración insuficiente de sueño durante los fines de semana, vigilias y baja calidad de sueño, así como color de piel negro o mulato con una duración insuficiente de sueño durante los días de clase, baja calidad del mismo y vigilias. Tener clases por la mañana se asoció a sueño insuficiente durante los días de clase, somnolencia diurna, y el sexo femenino con somnolencia, baja calidad y vigilias. Los disturbios de sueño más frecuentes fueron sueño insuficiente durante los días de clase, baja calidad autopercibida y somnolencia diurna. El consumo de alcohol y cigarrillos, así como el color negro o mulato fueron los principales factores asociados a los disturbios.

Trastornos del Sueño; Sueño; Somnolencia; Estudiantes
Recebido em 26/Abr/2019

Versão final reapresentada em 07/Ago/2019

Aprovado em 06/Set/2019 